Chapter 14

\title{
The Role of Epigenetics in Neurodegenerative Diseases
}

\author{
Luca Lovrečić, Aleš Maver, Maja Zadel and \\ Borut Peterlin
}

Additional information is available at the end of the chapter

http://dx.doi.org/10.5772/54744

\section{Introduction}

Neurodegenerative disorders are among the greatest challenges and among the most serious health problems that will have to be faced by the modern societies across the world, especially in light of increasing population age. They are incurable, progressive conditions resulting from continuous degeneration and death of nerve cells. Most of these disorders become more common with advancing age, including Alzheimer's disease and Parkinson's disease. The burden of these neurodegenerative diseases is growing inexorably as the population ages, with incalculable economic and human costs. According to a collaborative study of the World Health Organization, the World Bank and the Harvard School of Public Health (the Global Burden of Disease Study) dementia and other neurodegenerative diseases will be the eighth cause of disease burden for developed regions in 2020 [1, 2]. Moreover, they will become the world's second leading cause of death by 2050, overtaking cancer [2]. These future projections are of course only estimates, but they in combination with current state certainly support the fact that neurodegenerative diseases are of an increasing public concern

Although a minor subset of these disorders is caused by clearly defined genetic factors, for example Huntington's disease, the largest proportion arise due to a complex interplay of genetic and environmental factors. For this reason, delineation of specific risk factors, useful biomarkers, potential new therapeutic targets and agents and even definite diagnosis still remains difficult. Pathological characteristics in brain during the process of neurodegeneration show considerable overlap among different types of neurodegenerative cognitive and motor impairment [3]. Moreover, pathological findings do not neccessarily correlate with clinical findings, meaning that extensive neuropathology does not definitely imply a severly impared neurological function and, on the other hand, minor pathology may entail significantly impaired neurological function [4,5]. Namely, neuropathological processes are not necessarily 
the cause of the underlying disease at the early disease stages, but may merely be the reflection of fundamental disease processes, yet unknown in majority of neurodegenerative disorders. Later, as disease progresses, neuropathological changes probably contribute to disease progression in a positive feedback loop.

Currently, there is no diagnostic test that can clearly indicate the presence, absence, or category of a neurodegenerative disease. Individual diagnosis is based on clinical evaluation of the symptoms and specific neuroimaging characteristics, and it often takes years to make one. The exceptions are monogenic diseases, such as Huntington's disease (HD), where specific genetic test confirms the diagnosis.

Another unexplained field is underlying mechanism in a majority of neurodegenerative diseases. Most are characterized by the aggregation of intracellular proteins, but it is not clear whether this is a primary mechanism or a consequence of another disturbed cell function. The potential mechanisms of neurodegeneration are innumerable, including primary effects of protein homeostasis, disturbed protein degradation, gene expression, transcriptional regulation, mitochondrial dysfunction, etc. There is the urgent need to better understand disease pathophysiology in order to improve early diagnosis and development of disease-modifying treatments.

In the recent decade, however, a role of epigenetic alterations in development of neurodegenerative diseases has been increasingly discussed. Epigenetic constitution presents a landscape where environmental factors may interact with genetic make-up of an individual. Additionally, the development of high-throughput technologies for genomic, trancriptomic and epigenomic profiling now offers insight into epigenetic alterations in neurodegeneration, as well as opportunity for an integrative view of its interactions with various 'omic' levels. Interpretation of epigenomic profiling results in the context of neurodegeneration and the methodology for integration of heterogeneous 'omic' data opens an array of novel biological and bioinformatic challenges and requires the development of novel approaches towards analysis of these data.

\section{Neurodegenerative disorders}

\subsection{Common neurodegenerative disorders - Alzheimer and Parkinson disease}

Parkinson's disease (PD) and Alzheimer disease (AD) are two most common neurodegenerative diseases. Both are very heterogeneous in regard to the causes (combination of genetic and environmental factors), wide range in the age at onset, vast variety in leading symptoms and presenting clinical manifestations, disease progression and responses to different therapies. Definitive clinical diagnosis is hard, although a number of clinical and neuropsychological tests are often employed when making it. AD is detected with approximately $85-90 \%$ accuracy and PD with approximately 75\% accuracy. Despite endless number of research groups worldwide trying to uncover and explain the pathogenesis of both $\mathrm{AD}$ and $\mathrm{PD}$, they still remain unexplained. 
Ballard et all estimated in 2011[6] that 24 million people have dementia worldwide, majority attributable to AD. Namely, it is foreseen that more than 80 million people will have AD by 2040 [7], because of the population ageing and age-dependent incidence rate of AD. In order to improve development of disease-modifying treatment we need to understand the underlying pathophysiology of the disease. It is a progressive neurodegenerative disease which predominantly affects cortical and hippocampal neurons and leads to their irreversible loss [8]. Major clinical signs and symptoms include progressive impairment in memory, judgment, decision making, orientation to physical surroundings, and language. There are several neuropathological features in $\mathrm{AD}$, but only 2 are considered essential for the diagnosis - $\beta$ amyloid containing extracellular senile plaques and neurofibrillary tangles, composed of a hyperphosphorylated form of the microtubular protein tau. Others include synapse loss, neuron loss, atrophy, gliosis, degenerative changes in white matter, granulovacuolar degeneration, other protein aggregates [9].

PD was first described by James Parkinson in earyl 1800s as "shaking palsy". It is the second most prevalent neurodegenerative disease after AD. There are over 6 million people with PD worldwide (European Parkinson's Disease Association, EPDA). As elderly population increases, this estimate is predicted to double by the year 2040. The typical clinical signs are resting tremor, bradykinesia, muscle rigidity, and postural instability. The key neuropathological characteristics are the loss of neurons in the substantia nigra and the presence of neuronal protein aggregates termed Lewy bodies [10]. It is believed that approximately $5-20 \%$ of patients have monogenic PD with more than 10 genes being identified as causative. These gene defects in familial PD highlight the importance of genetic influences in this disease, however the majority of PD cases are considered sporadic and idiopathic nad thus believed to be largely influenced also by other factors. The current consensus suggests that PD develops from multiple risk factors including aging, genetic predisposition, and environmental exposure [11].

Important future challenge in the management $\mathrm{AD}$ and $\mathrm{PD}$ remains the establishment of early diagnosis or even identification of individuals prior to the onset of dementia in AD or resting tremor in PD. This implicates advancement in understanding disease pathogenesis and development of diagnostic approaches, including disease/process specific biomarkers.

\subsection{Huntington disease - A model of genetic neurodegenerative disorder}

Huntington disease is a late onset, autosomal dominant genetic disease - its cause is invariably trinucleotide expansion mutation, known for almost 2 decades [12]. Typical clinical signs are progressive motor impairment, cognitive decline and various psychiatric symptoms with the age of onset between 30 and 50 years. The disease is fatal after 15-20 years of progressive neurodegeneration [13]. So far, no effective treatment has been available to cure the disease or to even efficiently slow its progression. Although hyperkinesias and psychiatric symptoms respond well to pharmacotherapy, neuropsychological deficits and dementia remain untreatable [14]. There is no way of predicting the age at onset. Also, due to the insensitive clinical rating scales, it is not feasible to follow the disease progression over short time periods. Moreover, there are no specific measures to follow response to symptomatic treatment over 
short time periods. In addition, in the presymptomatic period when preventive treatment and slowing of neurodegeneration might be most effective, there are no measures/markers to monitor those responses and benefits.

The function of normal huntingtin in the cells and mutation mechanism that leads to neurodegeneration and typical signs are still not clear, despite the fact, that the responsible gene and mutation were already identified and characterized in 1993. Basic research created many different hypotheses about pathogenic mechanisms - they include disturbances in variety of biochemical pathways, such as accumulation of misfolded mutated proteins, apoptosis, protein degradation, intracellular signaling as well as oxidative stress, disturbed transcription regulation $[15,16]$ and epigenetic processes [17].

\section{Epigenetic mechanisms}

Increasing interest in epigenetics which is currently one of the most rapidly expanding fields in biomedical research has been accompanied by several breakthroughs like finding new histone variants and modifications, the discovery of the CpG island shores and most of all possibility of genome-wide analysis of epigenetic marks - epigenomic analysis - at single nucleotide resolution. The recognition of the role of epigenetics and how important it could be in human disease was first discussed in oncology and further extended to neurodevelopment and neurodegenerative diseases.

Changes in gene expression which are heritable are not always due to alterations in DNA sequence but are also attributable to epigenetic mechanisms. This could explain many cases where different phenotypes arise from the same genotype, such as monozygotic twins which are identical at DNA sequence level but have different DNA methylation and histone modification profiles [18] that possibly affect the penetrance for several complex diseases (cancer, autoimmune, neurodegenerative and cardiovascular diseases).

Studying epigenetic mechanisms made it possible to map epigenetic marks which are critical for regulating gene expression. The importance of epigenetics in maintaining normal development and in being one of the key factors in cellular differentiation can be explained by the observing how aberrant placement of epigenetic marks and mutations in the epigenetic machinery is involved in disease [19].

There are three distinct, yet highly interrelated, major mechanisms of epigenetic regulation (Figure 1) [20] :

1. DNA methylation refers to the addition of a methyl group to the 5-position of cytosine in the context of CPG dinucleotides to define the "fifth base of DNA."

2. Histone modification - The fundamental repeating unit of chromatin is the nucleosome comprised of an octamer of core histone proteins. Posttranslational modifications of the amino-terminal tails of histone proteins and the density of these proteins per unit length of DNA, can importantly affect chromatin structure and constitute a putative "histone code." 


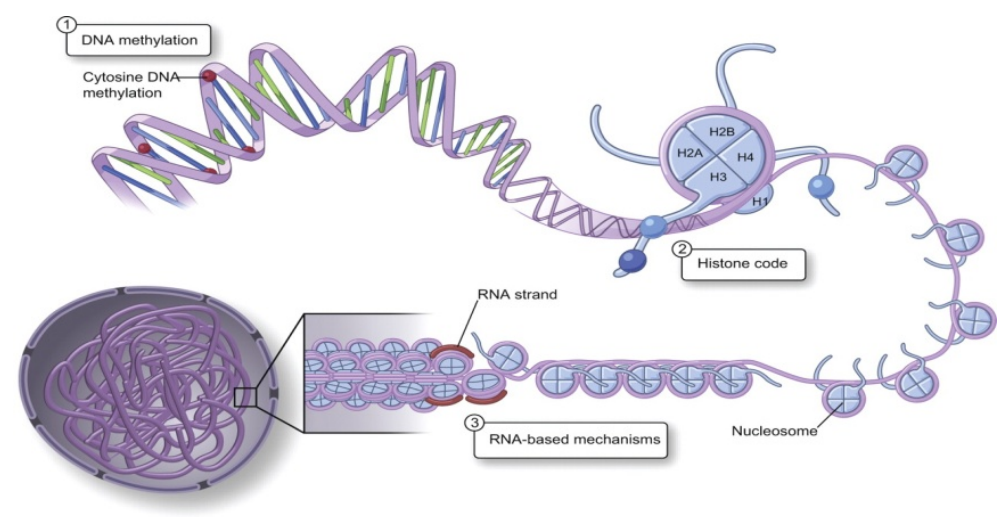

$\overline{\text { (Reproduced with }}$ permission, Yan, M. S. et al. J Appl Physiol 2010;109:916-926).

Figure 1. Schematic representation of 3 fundamental mechanisms of epigenetic gene regulation.

3. RNA-based mechanisms have also recently been shown to impact the higher-order structure of chromatin, such as small noncoding RNAs.

\subsection{DNA methylation}

This is the most widely studied epigenetic mechanism which consists of covalent addition of methyl group at the 5-position of cytosines followed by guanines (CpG dinucleotides) and is usually associated with gene silencing. $\mathrm{CpG}$ dinucleotides are generally clustered in regions called CpG islands defined as regions with G-C content of at least $50 \%$. Human gene promoters are associated with $\mathrm{CpG}$ island in about $60 \%$. In normal cells they are usually unmethylated and about $6 \%$ of them become methylated in a tissue-specific manner in differentiated tissue or during early development [21].

DNA methylation is not present only at CpG islands. Recently, a new term has been coined, $\mathrm{CpG}$ island shores which are regions of lower $\mathrm{CpG}$ density that are located close $(\approx 2 \mathrm{~kb})$ to the $\mathrm{CpG}$ islands. Additionally, it seems that methylation of $\mathrm{CpG}$ island shores is closely related to the inactivation of transcription and is also found that most of tissue-specific methylation of DNA occurs not at CpG islands but CpG island shores [22].

DNA methylation can inhibit gene expression in direct or indirect ways. Indirect transcription inhibition is mediated through promoting activation of methyl-CpG-binding domain (MBD) proteins by methylated DNA, which inhibit transcription [23]. Direct transcriptional inhibition is possible by interruption of activity of DNA binding proteins from their target sites.

A significant portion of methylated CpG islands is also found in repetitive elements. This methylation is functional and needed because of protection from reactivation of endoparasitic sequences which can cause chromosomal instability, gene disruption and translocations [24]. Eventhough DNA methylation occurs mostly in CpG islands in mammals, non-CG methylation has recently been described in humans at $\mathrm{CHG}$ and $\mathrm{CHH}$ sites $(\mathrm{H}=\mathrm{A}, \mathrm{C}$ or $\mathrm{T})$. The level of 
of non-CpG methylation decrease during differentiation and mechanisms of non-CpG methylation actually remains unclear at the moment [25].

Other epigenetic regulators linked to DNA methylation are DNA methylation enzyme DNA methyltransferases (DNMT) [26]. DNMT enzyme family mediates DNA methylation by catalyzing the transfer of a methyl group from an S-adenosyl-L-methionine to cytosine. In mammals there are five membrs of the DNMT family, but only three of them have methylatransferase activity - DNMT1, DNMT3a and DNMT3b.

The question that still remains unclear is how DNA methylation machinery is directed to specific sequences. Several proposed mechanisms imply there should be some interaction of DNMTs with other epigenetic factors.

\subsection{Histone modifications}

Another important epigenetic mark is histone modification. There are 4 groups of core histones $\mathrm{H} 2 \mathrm{~A}, \mathrm{H} 2 \mathrm{~B}, \mathrm{H} 3$ and $\mathrm{H} 4$ which form $\mathrm{H} 2 \mathrm{~A}$ and $\mathrm{H} 2 \mathrm{~B}$ dimers and one $\mathrm{H} 3-\mathrm{H} 4$ tetramer. Together they form nucleosome - fundamental unit of cromatin structure. These proteins provide a solid structure for DNA helix and also an interactive surface as N-terminal regions of histones protrude from the nucleosome and are susceptible to interactions with other proteins.

The residues at histone tails are subject to many post-translational modifications like acetylation, methylation, phosphorylation, SUMOylation and ADP-ribosylation [27]. Post-translational modifications are dynamic and reversible processes mediated by two antagonistic sets of enzyme-complexes that can attach or remove corresponding chemical groups. Acetylation at lysine residues is one of most thoroughly studied histone modifications and is associated with transcriptional activation [28]. Modifications are made by histone acetyl transferases (HATs) and can be reverted by histone deacetylase (HDACs).

As previously mentioned, there are interactions between all epigenetic marks. An interesting example of such interplay between histone modification and DNA methylation is relationship between DNA methyltransferase3 (DNMT3L) and H3K4 where DNMT3L specifically interacts with histone $\mathrm{H} 3$ tails inducing de novo DNA methylation [19].

\subsection{RNA-based mechanisms - Noncoding RNA-mediated modulation of gene expression}

RNA mediated post-transcriptional gene silencing (TGS) was first observed already in 1989 in tobacco plants [29] and later became known as an important epigenetic mechanism also in humans. It has also been shown in the last years that small RNAs are directed to the targeted promoter regions and this leads to modulation of silent state chromatin modifications [30]. In addition to gene silencing, there is evidence to suggest involvement of small RNAs in additional aspects of transcriptional regulation. These molecules may also activate certain genes, when targeted to promoter regions low in GC content [31]. One subtype of small RNAs are microRNAs, which excert their role on post-transcriptional level, probably by influencing the stability, compartmentalization and translation of mRNAs. In this way, expression of numerous genes is regulated related to different key biological processes cellular processes such as proliferation, morphogenesis, apoptosis and differentiation [32]. 
In addition to above described epigenetic mechanism, there is another mechanisms, tightly linked to both histone modifications and DNA methylation - nucleosome positioning.

Gene expression is also regulated by DNA packed into nucleosomes as these represent a barrier to transcription that blocks access of activators and transcription factors. For instance nucleosome displacement of about $30 \mathrm{bp}$ at transcription start site (TSS) has been reported which leads to changes in RNA polymerase II activity [19]. Gene activation is highly correlated with loss of nucleosome directly upstream of the TSS, whereas the occlusion of TSS by a nucleosome is correlated with gene repression [33]. Nucleosomes can be destabilized or ejected in ATP-hydrolysis dependent manner by groups of large macromolecular complexes, known as chromatin remodeling complexes. There are four families - SWI/SNF, ISWI, CHD and INO80 [34].

In general very little is known about specific mechanisms of nucleosome positioning and further investigation will give us more insights into these processes.

\section{Epigenetics and its role in neurodegeneration}

Proper differentiation and function of the central nervous system cells are subject to significant influence of a variety of epigenetic modifications. It has been shown that substantial reorganization of the epigenome on the level of cytosine methylation and histone modifications occurs throughout early brain development and continuing through the process of aging [35].

Most notably, the association between epigenetic signature and neurologic disease has been established through observation of monogenic neurodevelopmental disorders resulting from mutations in genes coding for proteins directly involved in core processes of methylation and histone modification. While mutatations in the MECP2 gene, coding for Methyl-CpG-binding protein and related to Rett syndrome, are probably most widely known for association of dysfunctional epigenetic regulation and neurologic disease, mutations in several similar genes have recently been related to neurologic disesease occuring in a variety of age groups.

The role of epigenetic alterations in common neurodegenerative disorders, such as Huntington's, Parkinson's and Alzheimer's disease, on the other hand, has not been considered until recently. Specific disturbed epigenetic mechanisms and changes in all three neurodegeneration NDG disorders are shown in Figure 2.

Surprisingly, it has been shown in the recent decade, that inhibition of histone deacetylase enzymes (HDACs) confers neuroprotective effects in invertebrate and mouse models of Huntington's disease [36]. It is thought that observed beneficial effects of HDAC inhibitors results from re-stabilization of gene transcription, owing to a shift of histone acetylation equilibrium towards increased acetylation of histones, relaxation of DNA-chromatin complexes and subsequent increase of gene transcription. In addition to putative alleviation of symptoms observed in Huntington's disease, the potential benefit of HDAC inhibitors has been considered in a range of neurologic diseases, from monogenic neurologic diseases to common neurodegenerative disorders, such as Parkinson's and Alzheimer's disease[35]. 


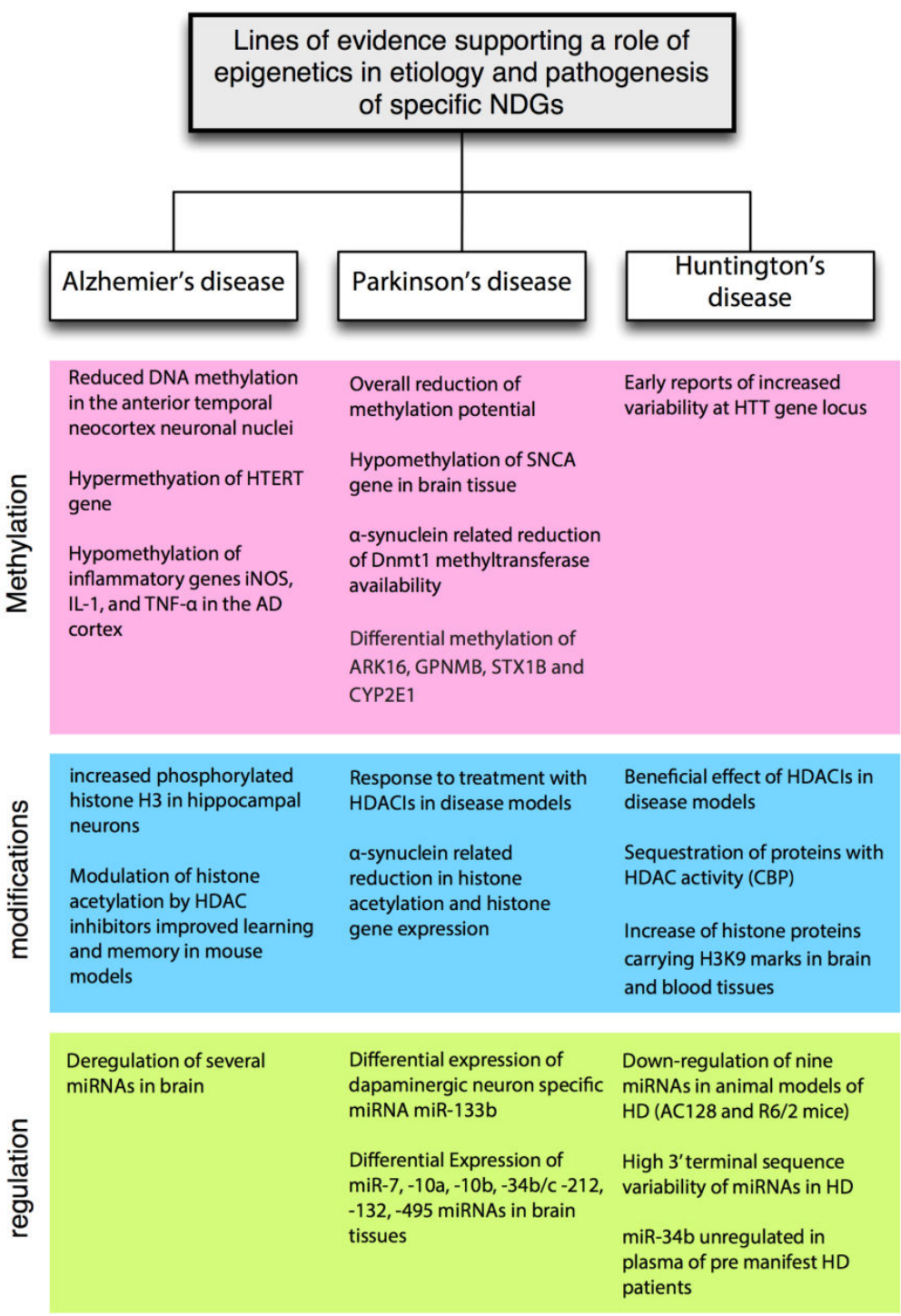

Figure 2. Key epigenetic changes in $P D, A D$ and $H D$

Other line of evidence stems from the fact that a multitude of monogenic disorders, resulting from mutations in genes essential for proper epigenetic regulation, are characterized by symptoms that arise late in the course of life, such as cerebellar ataxia and hereditary neuropathy. This observation rose a possibility that common neurodegenerative disorders may 
share pathogenetic mechanisms and be related to dysfunctions of methylation and histone modifications. It has been demonstrated that nuclei of brain cells from patients with Parkinson's disease contain reduced amounts of methyltransferase enzymes (most notably Dnmt1), leading to dysfunctional methylation of several genes playing a key role in PD pathogenesis, including synuclein- $\alpha$ gene, whose accumulation is observed in plaques of patients with PD [37]. It is also hypothesized that mitochondrial function, commonly perturbed in brain cells of patients with neurodegenerative disorders, is affected by methylation patterns of mitochondrial DNA [35].

Moreover, it was shown that protein aggregates constituting plaques found in brain cells of patients with Alzheimer's disease and Huntington's disease, contain sequestred proteins with histone acetyltranferase activity (notably CBP protein), potentially leading to reduced histone acetylation [38]. Increase of histone proteins carrying H3K9 marks has also been detected in blood and brain tissues of patients with Huntington's disease [39].

This novel evidence substantiates that epigenetic modifications may play a significant role in the etiology of neurodegenerative diseases and pathogenetic mechanisms involved in their propagation and may also present a great opportunity for therapeutic interventions.

\subsection{Epigenetics in Parkinson's disease}

Parkinson's disease (PD) is the second among most common neurodegenerative disorders and is characterized by progressive depletion of dopaminergic neurons within the substantia nigra, clinically manifesting as progressive symptoms of tremor, rigidity, bradykinesia and postural instability $[40,41]$. PD belongs to a group of complex diseases and is hypothesized to arise consequentially to interaction of a multitude of genetic and environmental factors. Details of specific sites of genetic predisposition and environmental insults, however, remain largely unclear [42]. Nevertheless, discovery of monogenic forms of PD provided a great insight into specific physiologic pathways that, once perturbed, lead to destruction of dopaminergic neurons. A significant proportion of cases with familial forms of PD, following clear Mendelian inheritance has been identified. Here, causative mutations have been discovered, offering valuable insight into intricacies and key points of disease pathogenesis. To date, mutations in genes SNCA (encoding $\alpha$-synuclein protein), PARK2 (parkin), PINK1 (PTEN-induced kinase protein 1), UCHL1 (ubiquitin carboxyl-terminal hydrolase isozyme L1), DJ1 (DJ-1 protein), and LRRK2 (leucine-rich repeat serine/threonine-protein kinase 2) were identified as a cause of familial PD [41].

Perhaps the most studied gene in light of potential epigenetic alterations in PD is SNCA gene. Depositions of misfolded $\alpha$-synuclein constitute a pathologic hallmark of Parkinson's disease (Lewy bodies) and co-locate with sites of neuronal loss. As $\alpha$-synuclein toxic effects are primarily exerted in neuronal nuclei, it has been hypothesized that it perturbs the distribution and organization of DNA and histone epigenetic modifications [43]. Indeed, recent studies have demonstrated $\alpha$-synuclein associates with histones and inhibits their acetylation, doing so through its association with Sirtuin-2 (Sirt2) histone deacetylase. Interestingly, in a Drosophila model of PD, targeted down-regulation of Sirt2 resulted in reduced $\alpha$-synuclein toxicity [44]. Additionally, other histonic modifications have been related to increased or 
decreased death of neurons in PD. Treatment with MPTP (1-methyl-4-phenyl-1,2,3,6-tetrahydropyridine), commonly used in animal models to provoke PD symptoms, has been assocated with increased presence of $\mathrm{H} 3$ histonic marks, which has been reverted after treatment with levo-dopa [45]. In a C. elegans model of PD, where overexpression of SNCA was stimulated, reduced expression of nine genes coding for histone proteins was observed [46]. In addition, a recent study has linked a mutation (A53T) in SNCA gene to monoallelic silencing of transcription form mutated allele, which was shown to result from histonic modifications at that site [47].

On the other hand, the role of DNA methylation in PD is currently unclear. It has been shown that metabolism of one-carbon compounds required for normal methylation is perturbed in PD [48]. Several studies have already pointed to the possibility of altered SNCA promoter gene methylation in relation to neuropsychiatric disorders [49]. Methylation of SNCA intron 1 has been associated with decrease in SNCA transcription and reduction of methylation at this site was observed at several brain regions affected in PD (including substantia nigra) [50]. This observation was further substantiated in analyses of other parts of the gene, where hypomethylation was also detected [51]. These results raise the possibility that increase in $\alpha$-synuclein production may result from increased SNCA expression, potentially as a consequence of reduced methylation status of this gene. Additionally, $\alpha$-synuclein has been shown to sequester DNA methyltransferase 1 (DNMT1), resulting in decreased overall methylation of genes in brain tissues of PD patients [37]. A study investigating larger set of genes, has revealed that other genes besides SNCA are characterized by differential methylation in PD (ARK16, GPNMB, and STX1B) [52]. Recently, these investigations have been expanded to global epigenomic scale of methylation in postmortem brain samples and a novel gene characterized by hypomethylation in PD was identified (CYP2E1, the cytochrome P450, family 2, subfamily E, polypeptide 1). In accordance, increased expression of the same gene has also been detected. Interestingly, CYP2E1 knock-out mice models have also been shown to be protected from MPTP toxic effects [53].

The role of miRNA regulatory system has also been implicated and surveyed in the context of PD. Initial study, performed by Kim et al, reported a detection of miRNA molecule (miR-133b) with expression specific to midbrain dopaminergic neurons and reduction of expression level in PD patients' midbrain tissue samples [54]. Differences in expression of several other microRNAs have been detected in early symptomatic mouse models of PD (miR-10a, -10b, -212, -132, -495) [55]. In addition, miR-7 has been found to repress expression of SNCA gene in two independent studies where it was demonstrated that its expression levels were reduced in MPTP cell culture and animal models $[56,57]$. A global miRNA profiling approach in PD brain samples revealed downregulation of $\mathrm{miR}-34 \mathrm{~b} / \mathrm{c}$, which has an important role in regulation of mitochondrial function. This deregulation was particularly notable in patients early in disease course, who have not yet been subjected to PD treatment modalities [58]. In another study, alterations of miRNAs could even be detected in blood samples from PD patients in regard to affection and pre- and post-treatment status. Their expression levels could be used to distinguish patients from controls based on blood expression profiles [59]. 
These lines of evidence provide support for the possibility that a proportion of pathways leading to PD could be better understood by incorporating epigenetic regulome into the our current disease models. In addition, epigenetic alterations provide novel treatment targets, allowing re-stabilization of perturbed cellular mechanisms. Most notably, histone deacetylase inhibitors (HDACIs), currently already approved for treatment of haematologic malignancies, have been shown to rescue $\alpha$-synuclein- induced toxicity in models in vitro and in vivo. Several new forms of HDACIs and DNA-demethylating drugs are currently in preclinical testing stage and provide an array of new opportunities to benefit patients with PD [60].

\subsection{Epigenetics in Alzheimer disease}

Alzheimer disease, as discussed above, is the leading couse of dementia world wide. It is characterized by dementia that typically begins with subtle and poorly recognized failure of memory and slowly becomes more severe and, eventually, incapacitating. Other findings include confusion, poor judgment, language disturbance, agitation, withdrawal, and hallucinations. As is true for $\mathrm{PD}, \mathrm{AD}$ is believed to be a complex disease, combining the effects of genetic and environmental factors. On the other hand, approximately $25 \%$ of all AD is familial, $95 \%$ of which is late-onset (age $>60-65$ years) and $5 \%$ is early-onset (age $<60$ years). In familial $\mathrm{AD}$ definite causative genes and mutations are known, offering important models for studying disease pathogenesis. To date, mutations in three genes are undoubtfully linked to AD Presenilin-1 (PSEN1), Presenilin-2 (PSEN2) and Amyloid beta A4 protein (APP). Others remain to be discovered. This is also true for the cause of sporadic $A D$, which can in part be attributable to epigenetic mechanisms.

Epigenetics is an emerging field in the light of potential impact on development of neurodegenerative diseases. It is a mechanism independant of DNA sequence with effects on gene transcription, yet in specific way it is heritable. It is well known that epigenetic modifications alter transcriptional activity of thousands of genes and simultaneously of many different cellular pathways in individually specific manner, dependant also on environmental factors and yet unknown co-factors. In this way epigenetics might constitute a crossroad for diverse pathophysiological mechanisms and risk factors contributing to development of Alzheimer disease. In addition, epigenetics may explain a part of mechanism by which AD in a first degree relative means an increased risk of "sporadic" disease, since epigenetic impressions are passed from generation to generation. Different mechanism have been implicated-DNA methylation, histone acetylation, miRNAs.

\subsubsection{DNA methylation in $A D$}

An important suggestive evidence of the role of epigenetic modifications in AD was a study on monozygotic twins discordant for AD, where status of DNA methylation in temporal neocortex neuronal nuclei was significantly altered in the AD twin [61].

It has been shown this year in genome-wide DNA methylation study that more than $900 \mathrm{CpG}$ sites representing 918 unique genes might be associated with late onset AD. The best candidate gene turned out to be a Transmembrane Protein 59 (TMEM59), whose promoter was hypo- 
methylated in AD [62]. Interestingly, TMEM59 was previously linked to amyloid precursor protein shedding, which is a central regulatory point in the production of amyloid $\beta$ peptid in $\mathrm{AD}$ [63]. Regulation of amyloid precursor protein shedding is still not clear and mostly unknown at the molecular level.

Another gene candidate shown to be differentially methylated is mRNA componentof telomerase HTERT (human telomerase reverse transcriptase), potentially involved in higher telomerase activity and immune dysfunctions in AD pathogenesis [64]. Disturbances in inflammatory regulation and response has been widely accepted and studied in AD for more than a decade. Numerous studies have proven this connection, for example, inflammatory mediators and signs of oxidative stress were described mostly in regions of beta-amyloid peptide deposits and neurofibrillary tangles [65]. Also, in vitro and in vivo studies on connection between pro-inflammatory cytokines and processing and production of the beta-amyloid peptid shown important influence [66]. In addition, whole genome expression studies showed widespread transcriptional alterations - decreased neurotrophic support and activated apoptotic and neuroinflammatory signaling in AD brain [67]. Specific studies on DNA methylation have added to this hypothesis by detecting hypomethylation of iNOS, IL-1, and TNF- $\alpha$ in the AD brain [65].

\subsubsection{Hystone modification in $A D$}

Another pathway of epigenetic regulation of transcription and other functions in cells is chromatin acetylation and deacetylation of histone proteins. Many studies associated histone hypoacetylation and transcriptional dysfunction with many different neurodegenerative conditions, including AD [68], Parkinson's disease [69] and Huntington's disease [70]. Direct evidence of disturbed histone modifications in $\mathrm{AD}$ was proposed in a study where elevated levels of phosphorylated histone $\mathrm{H} 3$ in AD hippocampal neurons were found and dislocalised to neuronal cytoplasm, as opposed to the nucleus as in actively dividing cells [71]. On the other hand, a recent study using novel proteomic approaches showed that histone acetylation is significantly lower in AD temporal lobe as compared to aged controls [72].

Using HDAC inhibitors to manipulate histone acetylation in several animal models of $\mathrm{AD}$ showed important potential of these molecules as treatment options. For example, valproic acid, which has HDAC1 inhibitor activity, decreased the production of amyloidbeta precursor protein and reduced plaque burden in the brains of Alzheimer's disease transgenic mouse model [73]. Another study reported beneficial role of phenylbuthyrate in cognitive impairment and neuropathology in AD. Using sodium 4-phenylbutyrate to treat $\operatorname{Tg} 2576$ mice it was shown that it reversed spatial memory loss and normalized levels of phosphorylated tau in the hippocampus. At the same time, it failed to change amyloid-beta precursor level [74]. This is in concordance with earlier studies which have shown that changes in amyloid-beta precursor levels are not necessarily associated with improvement in learning and memory performance [75, 76].

Another important finding was that increased histone acetylation induced sprouting of dendrites, increased number of synapses and reinstated learning behaviour and access to long-term memories, after already present brain atrophy and neuronal loss in a mouse 
model [77]. The author suggested that histone deacetylase inhibitors might be potential therapeutic agents for $\mathrm{AD}$ nad other neurodegenerative diseases where learning and memory impairment is present.

As it seems, histone modifications happen and have an important role in AD and AD animal models. Still, the underlying mechanisms and regulation and post-modification pathways are complex and not fully understood yet.

\subsection{3. $\operatorname{miRNA}$ in $A D$}

Micro RNAs (miRNAs) are post-transcriptional regulators that bind to complementary sequences on target mRNAs. This usually results in translational repression or target degradation and gene silencing. It is assumed that human genome might encode approximatelly 1000 miRNAs. miRNAs have been linked to posttranscriptional control of amyloid precursor protein expression [78], namely negative regulatory control by miR-101 and miR-16 [79]. The study clearly demonstrated that amyloid precursor protein is a target of miR-16 and that abnormally low expression of miR-16 might directly lead to protein accumulation in AD mice. Also, miRNAs are equally involved in the regulation of neuronal mRNA alternative splicing [80]. Smith et al have shown that miR-124 is down-regulated in AD brain and the same time, its underexpression in neurons is associated with inclusion of exons 7 and 8 to amyloid precursor protein. To contribute to this finding, they have also shown that ectopic expression of miR-124 reversed these effects in cultured neurons.

At present, miRNAs are hot topic and the full complement of miRNA that participate in the regulation of precursor protein expression, splicing and potential other steps in its accumulation are yet to be discovered.

\subsection{Epigenetics in Huntington's disease}

Huntington's disease (HD) is caused by dominant mutation with expanded number of glutamine codons within an existing poly-glutamine (polyQ) repeat sequence of a gene encoding for protein huntingtin (htt). Normal htt has 36 polyQ repeats and aberrant has more than 39 polyQ repeats. In case of 36-39 polyQ repeats we have an "intermediate allele" with incomplete penetrance and greater probability of becoming pathogenic if there is family history of Huntington's disease.

It has been shown in brain of mouse models [16] and in blood and brain of patients with HD that changes in gene expression occur very early in the disease course [81]. Gene transcription is regulated by complex interplay of different protein complexes, including transcription factors and histones, which in turn are regulated by covalent modifications such as acetylation, methylation and phosphorylation. Histone acetylation, which promotes transcription, is reduced in models of HD [82] and, on the other hand, histone methylation, which inhibits transcription, is increased [83]. Moreover, histone methylation was shown to be increased in HD patients, suggesting important role in disease pathogenesis [39].

Further evidence was provided by the use of histone acetylase inhibitors (HDACi), sodium butyrate and phenylbutyrate which increased survival of R6/2 mice. Also, improved motor 
performance and to some extend reversed alteration in gene expression and hypoacetylation at selected promoters in cerebellum of R6/2 mice was reported [82]. The exact mode of histone acetylation and methylation in HD is still unknown, but HDACs might be a potentially promissing treatment. One of HDAC inhibitors, sodium phenylbutyrate, is already approved in the treatment of urea cycle disorders and is under investigation for treatment of various refractory cancers, amyotrophic lateral sclerosis and spinobulbar muscular atrophy. A dosefinding study of this compound in human HD has already been conducted to determine the tolerability of the compound in patients with HD [84].

Another epigenetic mechanism, implicated in HD, is also disturbed miRNAs levels. Several miRNAs are significantly misregulated in HD brains compared with healthy controls. Five miRNAs, miR-9, miR-9*, miR-29b, miR-124a and miR-132 are significantly disregulated in HD cortex [85]. Interestingly, miR-9/miR9* are important in regulation of transcriptional repressor REST, which is mislocalized in brains neurons of patients with HD [86]. Together with other transcriptional factors it acts to regulate neuronal gene expression. How this is related to HD progression and potential for treatment remains yet to be elucidated.

In addition to human HD, an important role of miRNAs has also been shown in cell models of $\mathrm{HD}$ and linked directly to mutated protein huntingtin. Huntingtin has been shown to be the target of several miRNAs, miR-214, miR-150, miR-146a and miR-125b. Their expression reduced the expression of mutated protein. This regulation of huntingtin by miRNAs might provide a new approach to modulate HD [87].

\section{Conclusion}

Neurodegenerative diseases are becoming one of the most important public health issue in the developed world due to the population ageing. It is of paramount importance to improve our knowledge on pathophysiology of the group of these diseases with the aim to improve diagnostics, development of disease-modifying treatment and preventive measures. The most important field is early diagnosis with accurate risk estimates and potential preventive treatment. Numerous research groups and initiatives are working with this same goal in order to improve above mentioned issues.

Global epigenetic changes, with downstream effect on numerous genes and different biological pathways, appear to be involved in synchronous cellular response and alterations that direct development, aging, and, in some cases, even disease. Epigenetic modifications in the pathopsysiology of neurodegenerative diseases are lately becoming more and more important and widely discussed. This field is expected to provide important molecular mechanisms that will contribute to understanding of the pathogenesis, treatment response and even development of new therapeutic targets in the field of neurodegenerative disorders.

DNA methylation, histone modifications and small noncoding RNA regulation are different epigenetic mechanisms directly or indirectly linked to transcriptional activity and posttranslational modifications, such as alternative splicing. It has already been proposed and well 
accepted that these epigenetic alterations with consequent transcriptional dysregulation might be an important marker of disease status and its progression in many neurodegenerative diseases. The disruption of normal transcriptional pathways through altered epigenetic status triggers signaling cascades linked with a number of pathological mechanisms.

In this chapter we have shortly reviewed epigenetic role in Parkinson's disease, Alzheimer disease and Huntington's diseases. Epigenetic characteristics show an important role and certain specifics in all three. Moreover, the epigenetic modifications that have been reported in $\mathrm{PD}$ and $\mathrm{AD}$, are in some points similar to trends in normal aging. Therefore, in addition to giving us insight to pathogenesis of neurodegeneration, they might also explain why aging by itself is a risk factor for developing neurodegenerative disorder.

Instead of studying preselected genes or regions and their epigenetic characteristics, wholegenome epigenetic status, so-called epigenome should be studied. With the knowledge on direct impact of epigenetic to transcriptional dysregulation, it would be of great importance to conduct epigenomic studies in tandem with transcriptomic (genome-wide gene expression profiling) studies. Only with this approach is one able to directly validate functional effects of epigenetic changes on gene expression. True, research on the epigenetic components and mechanisms associated with neurodegeneration is still in its beginning and future will provide additional essential information.

Last but not least - epigenetic modification is a reversible characteristic while genetic mutation is not. Influencing and tightly regulating epigenetic modification is therefore theoretically very promising candidate method from a therapeutic perspective of neurodegenerative diseases. It is well known that certain therapeutic compounds can influence and change the DNA methylation status and transcriptional activity accordingly. Though, one has to bear in mind, that in general, epigenetic mechanisms exert effects on many genes simultaneously and the same is also true for currently known effective epigenetic therapeutics.

\section{Author details}

Luca Lovrečić*, Aleš Maver, Maja Zadel and Borut Peterlin

*Address all correspondence to: lucalovrecic@gmail.com

Clinical Institute of Medical Genetics, University Medical Center Ljubljana, Slovenia

\section{References}

[1] C.J.L. Murray, A.D.L., The Global Burden of Disease, 1996, World Health Organisation: Geneva. 
[2] Menken, M., T.L. Munsat, and J.F. Toole, The global burden of disease study: implications for neurology. Arch Neurol, 2000. 57(3): p. 418-20.

[3] Schneider, J.A., et al., Mixed brain pathologies account for most dementia cases in community-dwelling older persons. Neurology, 2007. 69(24): p. 2197-204.

[4] Pathological correlates of late-onset dementia in a multicentre, community-based population in England and Wales. Neuropathology Group of the Medical Research Council Cognitive Function and Ageing Study (MRC CFAS). Lancet, 2001. 357(9251): p. 169-75.

[5] Prohovnik, I., et al., Dissociation of neuropathology from severity of dementia in late-onset Alzheimer disease. Neurology, 2006. 66(1): p. 49-55.

[6] Ballard, C., et al., Alzheimer's disease. Lancet. 377(9770): p. 1019-31.

[7] Forlenza, O.V., B.S. Diniz, and W.F. Gattaz, Diagnosis and biomarkers of predementia in Alzheimer's disease. BMC Med. 8: p. 89.

[8] McKhann, G., et al., Clinical diagnosis of Alzheimer's disease: report of the NINCDS-ADR$D A$ Work Group under the auspices of Department of Health and Human Services Task Force on Alzheimer's Disease. Neurology, 1984. 34(7): p. 939-44.

[9] Hyman, B.T., et al., National Institute on Aging-Alzheimer's Association guidelines for the neuropathologic assessment of Alzheimer's disease. Alzheimers Dement, 2012. 8(1): p. $1-13$.

[10] Spillantini, M.G., et al., Alpha-synuclein in Lewy bodies. Nature, 1997. 388(6645): p. 839-40.

[11] Gao, H.M. and J.S. Hong, Gene-environment interactions: key to unraveling the mystery of Parkinson's disease. Prog Neurobiol, 2011. 94(1): p. 1-19.

[12] A novel gene containing a trinucleotide repeat that is expanded and unstable on Huntington's disease chromosomes. The Huntington's Disease Collaborative Research Group. Cell, 1993. 72(6): p. 971-83.

[13] Harper, P.S., Huntington's disease. Major Problems in Neurology1996, London: W.B. Saunders Company Ltd.

[14] Bonelli, R.M. and P. Hofmann, A systematic review of the treatment studies in Huntington's disease since 1990. Expert Opin Pharmacother, 2007. 8(2): p. 141-53.

[15] Harjes, P. and E.E. Wanker, The hunt for huntingtin function: interaction partners tell many different stories. Trends Biochem Sci, 2003. 28(8): p. 425-33.

[16] Sugars, K.L. and D.C. Rubinsztein, Transcriptional abnormalities in Huntington disease. Trends Genet, 2003. 19(5): p. 233-8.

[17] He, F. and P.K. Todd, Epigenetics in nucleotide repeat expansion disorders. Semin Neurol, 2011. 31(5): p. 470-83. 
[18] Kaminsky, Z.A., et al., DNA methylation profiles in monozygotic and dizygotic twins. Nat Genet, 2009. 41(2): p. 240-5.

[19] Portela, A. and M. Esteller, Epigenetic modifications and human disease. Nat Biotechnol, 2010. 28(10): p. 1057-68.

[20] Yan, M.S., C.C. Matouk, and P.A. Marsden, Epigenetics of the vascular endothelium. J Appl Physiol, 2010. 109(3): p. 916-26.

[21] Straussman, R., et al., Developmental programming of $C p G$ island methylation profiles in the human genome. Nat Struct Mol Biol, 2009. 16(5): p. 564-71.

[22] Irizarry, R.A., et al., The human colon cancer methylome shows similar hypo- and hypermethylation at conserved tissue-specific CpG island shores. Nat Genet, 2009. 41(2): p. 178-86.

[23] Esteller, M., Epigenetic gene silencing in cancer: the DNA hypermethylome. Hum Mol Genet, 2007. 16 Spec No 1: p. R50-9.

[24] Esteller, M., Cancer epigenomics: DNA methylomes and histone-modification maps. Nat Rev Genet, 2007. 8(4): p. 286-98.

[25] Laurent, L., et al., Dynamic changes in the human methylome during differentiation. Genome Res, 2010. 20(3): p. 320-31.

[26] Espada, J., et al., Epigenetic disruption of ribosomal RNA genes and nucleolar architecture in DNA methyltransferase 1 (Dnmt1) deficient cells. Nucleic Acids Res, 2007. 35(7): p. 2191-8.

[27] Kouzarides, T., Chromatin modifications and their function. Cell, 2007. 128(4): p. 693-705.

[28] Santos-Rosa, H., et al., Histone H3 tail clipping regulates gene expression. Nat Struct Mol Biol, 2009. 16(1): p. 17-22.

[29] Matzke, M.A., et al., Reversible methylation and inactivation of marker genes in sequentially transformed tobacco plants. EMBO J, 1989. 8(3): p. 643-9.

[30] Kim, D.H., et al., Argonaute-1 directs siRNA-mediated transcriptional gene silencing in human cells. Nat Struct Mol Biol, 2006. 13(9): p. 793-7.

[31] Janowski, B.A., et al., Activating gene expression in mammalian cells with promoter-targeted duplex RNAs. Nat Chem Biol, 2007. 3(3): p. 166-73.

[32] Carthew, R.W., Gene regulation by microRNAs. Curr Opin Genet Dev, 2006. 16(2): p. 203-8.

[33] Schones, D.E., et al., Dynamic regulation of nucleosome positioning in the human genome. Cell, 2008. 132(5): p. 887-98.

[34] Ho, L. and G.R. Crabtree, Chromatin remodelling during development. Nature, 2010. 463(7280): p. 474-84. 
[35] Jakovcevski, M. and S. Akbarian, Epigenetic mechanisms in neurological disease. Nat Med, 2012. 18(8): p. 1194-204.

[36] Steffan, J.S., et al., Histone deacetylase inhibitors arrest polyglutamine-dependent neurodegeneration in Drosophila. Nature, 2001. 413(6857): p. 739-43.

[37] Desplats, P., et al., Alpha-synuclein sequesters Dnmt1 from the nucleus: a novel mechanism for epigenetic alterations in Lewy body diseases. J Biol Chem, 2011. 286(11): p. 9031-7.

[38] Nucifora, F.C., Jr., et al., Interference by huntingtin and atrophin-1 with cbp-mediated transcription leading to cellular toxicity. Science, 2001. 291(5512): p. 2423-8.

[39] Ryu, H., et al., ESET/SETDB1 gene expression and histone H3 (K9) trimethylation in Huntington's disease. Proc Natl Acad Sci U S A, 2006. 103(50): p. 19176-81.

[40] Lees, A.J., J. Hardy, and T. Revesz, Parkinson's disease. Lancet, 2009. 373(9680): p. 2055-66.

[41] Mayeux, R., Epidemiology of neurodegeneration. Annu Rev Neurosci, 2003. 26: p. 81-104.

[42] Klein, C. and A. Westenberger, Genetics of Parkinson's disease. Cold Spring Harb Perspect Med, 2012. 2(1): p. a008888.

[43] Kontopoulos, E., J.D. Parvin, and M.B. Feany, Alpha-synuclein acts in the nucleus to inhibit histone acetylation and promote neurotoxicity. Hum Mol Genet, 2006. 15(20): p. 3012-23.

[44] Outeiro, T.F., et al., Sirtuin 2 inhibitors rescue alpha-synuclein-mediated toxicity in models of Parkinson's disease. Science, 2007. 317(5837): p. 516-9.

[45] Nicholas, A.P., et al., Striatal histone modifications in models of levodopa-induced dyskinesia. J Neurochem, 2008. 106(1): p. 486-94.

[46] Vartiainen, S., et al., Identification of gene expression changes in transgenic C. elegans overexpressing human alpha-synuclein. Neurobiol Dis, 2006. 22(3): p. 477-86.

[47] Voutsinas, G.E., et al., Allelic imbalance of expression and epigenetic regulation within the alpha-synuclein wild-type and p.Ala53Thr alleles in Parkinson disease. Hum Mutat, 2010. 31(6): p. 685-91.

[48] Obeid, R., et al., Methylation status and neurodegenerative markers in Parkinson disease. Clin Chem, 2009. 55(10): p. 1852-60.

[49] Qureshi, I.A. and M.F. Mehler, Advances in epigenetics and epigenomics for neurodegenerative diseases. Curr Neurol Neurosci Rep, 2011. 11(5): p. 464-73.

[50] Jowaed, A., et al., Methylation regulates alpha-synuclein expression and is decreased in Parkinson's disease patients' brains. J Neurosci, 2010. 30(18): p. 6355-9.

[51] Matsumoto, L., et al., CpG demethylation enhances alpha-synuclein expression and affects the pathogenesis of Parkinson's disease. PLoS One, 2010. 5(11): p. e15522. 
[52] A two-stage meta-analysis identifies several new loci for Parkinson's disease. PLoS Genet, 2011. 7(6): p. e1002142.

[53] Kaut, O., I. Schmitt, and U. Wullner, Genome-scale methylation analysis of Parkinson's disease patients' brains reveals DNA hypomethylation and increased mRNA expression of cytochrome P450 2E1. Neurogenetics, 2012. 13(1): p. 87-91.

[54] Kim, J., et al., A MicroRNA feedback circuit in midbrain dopamine neurons. Science, 2007. 317(5842): p. 1220-4.

[55] Gillardon, F., et al., MicroRNA and proteome expression profiling in early-symptomatic alpha-synuclein(A30P)-transgenic mice. Proteomics Clin Appl, 2008. 2(5): p. 697-705.

[56] Junn, E., et al., Repression of alpha-synuclein expression and toxicity by microRNA-7. Proc Natl Acad Sci U S A, 2009. 106(31): p. 13052-7.

[57] Doxakis, E., Post-transcriptional regulation of alpha-synuclein expression by mir-7 and mir-153. J Biol Chem, 2010. 285(17): p. 12726-34.

[58] Minones-Moyano, E., et al., MicroRNA profiling of Parkinson's disease brains identifies early downregulation of miR-34b/c which modulate mitochondrial function. Hum Mol Genet, 2011. 20(15): p. 3067-78.

[59] Margis, R. and C.R. Rieder, Identification of blood microRNAs associated to Parkinsonis disease. J Biotechnol, 2011. 152(3): p. 96-101.

[60] Kazantsev, A.G. and L.M. Thompson, Therapeutic application of histone deacetylase inhibitors for central nervous system disorders. Nat Rev Drug Discov, 2008. 7(10): p. 854-68.

[61] Mastroeni, D., et al., Epigenetic differences in cortical neurons from a pair of monozygotic twins discordant for Alzheimer's disease. PLoS One, 2009. 4(8): p. e6617.

[62] Bakulski, K.M., et al., Genome-wide DNA methylation differences between late-onset Alzheimer's disease and cognitively normal controls in human frontal cortex. J Alzheimers Dis, 2012. 29(3): p. 571-88.

[63] Selkoe, D.J. and D. Schenk, Alzheimer's disease: molecular understanding predicts amyloid-based therapeutics. Annu Rev Pharmacol Toxicol, 2003. 43: p. 545-84.

[64] Silva, P.N., et al., Promoter methylation analysis of SIRT3, SMARCA5, HTERT and CDH1 genes in aging and Alzheimer's disease. J Alzheimers Dis, 2008. 13(2): p. 173-6.

[65] Akiyama, H., et al., Inflammation and Alzheimer's disease. Neurobiol Aging, 2000. 21(3): p. 383-421.

[66] Blasko, I., et al., TNFalpha plus IFNgamma induce the production of Alzheimer beta-amyloid peptides and decrease the secretion of APPs. FASEB J, 1999. 13(1): p. 63-8.

[67] Colangelo, V., et al., Gene expression profiling of 12633 genes in Alzheimer hippocampal CA1: transcription and neurotrophic factor down-regulation and up-regulation of apoptotic and pro-inflammatory signaling. J Neurosci Res, 2002. 70(3): p. 462-73. 
[68] Stilling, R.M. and A. Fischer, The role of histone acetylation in age-associated memory impairment and Alzheimer's disease. Neurobiol Learn Mem, 2011. 96(1): p. 19-26.

[69] Chuang, D.M., et al., Multiple roles of HDAC inhibition in neurodegenerative conditions. Trends Neurosci, 2009. 32(11): p. 591-601.

[70] Sadri-Vakili, G. and J.H. Cha, Mechanisms of disease: Histone modifications in Huntington's disease. Nat Clin Pract Neurol, 2006. 2(6): p. 330-8.

[71] Ogawa, O., et al., Ectopic localization of phosphorylated histone H3 in Alzheimer's disease: a mitotic catastrophe? Acta Neuropathol, 2003. 105(5): p. 524-8.

[72] Zhang, K., et al., Targeted proteomics for quantification of histone acetylation in Alzheimer's disease. Proteomics, 2012. 12(8): p. 1261-8.

[73] Su, Y., et al., Lithium, a common drug for bipolar disorder treatment, regulates amyloid-beta precursor protein processing. Biochemistry, 2004. 43(22): p. 6899-908.

[74] Ricobaraza, A., et al., Phenylbutyrate ameliorates cognitive deficit and reduces tau pathology in an Alzheimer's disease mouse model. Neuropsychopharmacology, 2009. 34(7): p. 1721-32.

[75] Malm, T.M., et al., Pyrrolidine dithiocarbamate activates Akt and improves spatial learning in APP/PS1 mice without affecting beta-amyloid burden. J Neurosci, 2007. 27(14): p. 3712-21.

[76] Dodart, J.C., et al., Immunization reverses memory deficits without reducing brain Abeta burden in Alzheimer's disease model. Nat Neurosci, 2002. 5(5): p. 452-7.

[77] Fischer, A., et al., Recovery of learning and memory is associated with chromatin remodelling. Nature, 2007. 447(7141): p. 178-82.

[78] Long, J.M. and D.K. Lahiri, Current drug targets for modulating Alzheimer's amyloid precursor protein: role of specific micro-RNA species. Curr Med Chem, 2011. 18(22): p. 3314-21.

[79] Liu, W., et al., MicroRNA-16 targets amyloid precursor protein to potentially modulate Alzheimer's-associated pathogenesis in SAMP8 mice. Neurobiol Aging, 2012. 33(3): p. 522-34.

[80] Smith, P., et al., In vivo regulation of amyloid precursor protein neuronal splicing by microRNAs. J Neurochem, 2011. 116(2): p. 240-7.

[81] Borovecki, F., et al., Genome-wide expression profiling of human blood reveals biomarkers for Huntington's disease. Proc Natl Acad Sci U S A, 2005. 102(31): p. 11023-8.

[82] Ferrante, R.J., et al., Histone deacetylase inhibition by sodium butyrate chemotherapy ameliorates the neurodegenerative phenotype in Huntington's disease mice. J Neurosci, 2003. 23(28): p. 9418-27. 
[83] Gardian, G., et al., Neuroprotective effects of phenylbutyrate in the N171-82Q transgenic mouse model of Huntington's disease. J Biol Chem, 2005. 280(1): p. 556-63.

[84] Hogarth, P., L. Lovrecic, and D. Krainc, Sodium phenylbutyrate in Huntington's disease: a dose-finding study. Mov Disord, 2007. 22(13): p. 1962-4.

[85] Packer, A.N., et al., The bifunctional microRNA miR-9/miR-9* regulates REST and CoREST and is downregulated in Huntington's disease. J Neurosci, 2008. 28(53): p. 14341-6.

[86] Zuccato, C., et al., Widespread disruption of repressor element-1 silencing transcription factor/neuron-restrictive silencer factor occupancy at its target genes in Huntington's disease. J Neurosci, 2007. 27(26): p. 6972-83.

[87] Sinha, M., J. Ghose, and N.P. Bhattarcharyya, Micro RNA -214,-150,-146a and-125b target Huntingtin gene. RNA Biol, 2011. 8(6): p. 1005-21. 
